\title{
Microsurgery for cerebral arteriovenous malformations: postoperative outcomes and predictors of complications in 264 cases
}

\author{
Thana Theofanis, M.D., ${ }^{1}$ Nohra Chalouhi, M.D., ${ }^{1}$ Richard Dalyai, M.D., ${ }^{1}$ \\ Robert M. Starke, M.D., M.Sc., ${ }^{1,2}$ Pascal Jabbour, M.D., ${ }^{1}$ \\ Robert H. Rosenwasser, M.D., ${ }^{1}$ and Stavropoula TJoumakaris, M.D. ${ }^{1}$ \\ ${ }^{1}$ Department of Neurosurgery, Thomas Jefferson University and Jefferson Hospital for Neuroscience, \\ Philadelphia, Pennsylvania; and ${ }^{2}$ Department of Neurological Surgery, University of Virginia Health System, \\ Charlottesville, Virginia
}

\begin{abstract}
Object. The authors conducted a study to assess the safety and efficacy of microsurgical resection of arteriovenous malformations (AVMs) and determine predictors of complications.

Methods. A total of 264 patients with cerebral AVMs were treated with microsurgical resection between 1994 and 2010 at the Jefferson Hospital for Neuroscience. A review of patient data was performed, including initial hemorrhage, clinical presentation, Spetzler-Martin (SM) grade, treatment modalities, clinical outcomes, and obliteration rates. Univariate and multivariate analyses were used to determine predictors of operative complications.

Results. Of the 264 patients treated with microsurgery, $120(45 \%)$ patients initially presented with hemorrhage. There were 27 SM Grade I lesions (10.2\%), 101 Grade II lesions (38.3\%), 96 Grade III lesions (36.4\%), 31 Grade IV lesions $(11.7 \%)$, and 9 Grade V lesions (3.4\%). Among these patients, $102(38.6 \%)$ had undergone prior endovascular embolization. In all patients, resection resulted in complete obliteration of the AVM. Complications occurred in 19 $(7.2 \%)$ patients and resulted in permanent neurological deficits in $5(1.9 \%)$. In multivariate analysis, predictors of complications were increasing AVM size (OR 3.2, 95\% CI 1.5-6.6; p = 0.001), increasing number of embolizations (OR 1.6, 95\% CI 1.1-2.2; $\mathrm{p}=0.01$ ), and unruptured AVMs (OR 2.7,95\% CI 1-7.2; $\mathrm{p}=0.05$ ).

Conclusions. Microsurgical resection of AVMs is highly efficient and can be undertaken with low rates of morbidity at high-volume neurovascular centers. Unruptured and larger AVMs were associated with higher complication rates.

(http://thejns.org/doi/abs/10.3171/2014.7.FOCUS14160)
\end{abstract}

$\begin{array}{llll}\text { KEY WORDS } & \text { arteriovenous malformation } & \text { microsurgery } & \text { m } \\ \text { embolization } & \text { endovascular } & \text { - Spetzler-Martin grade }\end{array}$

$\mathrm{C}$ EREBRAL arteriovenous malformations (AVMs) are vascular lesions composed of tortuous arteries and veins without intervening capillaries. It has been estimated that AVMs are found in approximately $0.1 \%$ of the US population and are responsible for approximately $2 \%$ of all hemorrhagic strokes. ${ }^{2.5}$ AVMs have an annual risk of intracerebral hemorrhage that varies anywhere from $1 \%$ to $25 \% .^{2,5,15}$ While cerebral AVMs are a relatively infrequent cause of stroke overall, they make up a particularly high percentage of hemorrhagic strokes in patients younger than 40 years. ${ }^{27}$ When symptomatic, AVMs typically present before the age of 40 years, with the most common clinical presentation being intraparenchymal hemorrhage. Meyer and colleagues ${ }^{16}$ have shown that cerebrovascular adaptation by capillary recruitment

Abbreviations used in this paper: $\mathrm{AVM}=$ arteriovenous malformation; $\mathrm{mRS}=$ modified Rankin Scale; $\mathrm{SM}=$ Spetzler-Martin. keeps cerebral blood flow at normal levels in the majority of cases and that chronic noninfarctional cerebral hypoperfusion is eventually the equivalent of physiological steal around AVMs ${ }^{16}$ which can result in another common clinical presentation, seizures. Also, due to the high risk of rupture in select AVM patients, intervention is an appropriate option. ${ }^{10,29}$

Prior to the era of microsurgical techniques, AVM resection was associated with significant morbidity and mortality. Complete cure of AVMs is an outcome that has become much more attainable over the past decades. Microsurgical excision remains the best option in select patients due to the lack of a latency period for hemorrhage and low risk of future recurrence. ${ }^{7,22,30}$ Investigators in the recently published ARUBA (A Randomized Trial of Unruptured Brain Arteriovenous Malformations) trial ${ }^{17}$ concluded that medical management of AVMs is superior to intervention. Since a mere 5 patients in the treatment arm received open surgery-the only curative treatment 
for AVMs-it is questionable whether one can draw any meaningful conclusions from the results of the ARUBA trial, which was stopped prematurely after a safety analysis. However, microsurgical outcomes are arguably one of the biggest aspects lacking from the ARUBA trial. ${ }^{17}$

The series presented here is a cohort of AVM patients, who were treated surgically at a single institution over the past 2 decades, among a larger number of patients who received varied treatments. The aim of this study was to reaffirm the safety and efficacy of microsurgical resection of cerebral AVMs in the wake of the highly criticized findings of the ARUBA trial. We also determined predictors of postoperative complications.

\section{Methods \\ Patient Selection and Characteristics}

Institutional review board approval was obtained prior to data collection. We searched our prospectively maintained database for all patients with AVMs who underwent microsurgical resection at our institution between 1994 and 2010. A total of 264 patients were identified and constituted our study population. During the same period of time, 774 patients with cerebral AVMs were treated at our institution. Medical charts, imaging studies, and follow-up notes were reviewed to determine initial hemorrhage, clinical presentation, treatment modalities, clinical outcomes, and obliteration rates. A Spetzler-Martin (SM) grade was assigned and documented by the attending neurosurgeon based on imaging findings.

\section{Outcome Measures}

All patients underwent a neurological examination that was documented by a physician on the neurosurgical team both preoperatively and each postoperative day until discharge from the hospital. Neurological outcomes are stratified according to the modified Rankin Scale (mRS) score, which was determined based on documented clinical findings upon arrival at the hospital and at the time of hospital discharge.

\section{Surgical Techniques}

For each patient, the process of selecting the best therapy included case review and treatment by a doctor trained in microsurgery, endovascular therapy, and radiosurgery. A treatment decision was reached based on both physician recommendations and patient preference, with the ultimate goal of permanently eliminating the AVM and preserving full neurological function and/or reversing deficits. Assessment of AVM obliteration was determined by intraoperative angiography.

A portion of patients received endovascular embolization prior to microsurgery. This procedure was performed via the transfemoral approach under monitored anesthetic care. The biological agents used were $N$-butyl2-cyanoacrylate (Codman Neurovascular) or Onyx (eV3). Each embolization procedure was performed with the intention of reducing blood flow to the AVM incrementally over time. Some patients received this procedure as a preoperative measure. However, for other patients, it was not until serial embolizations and the appropriate followup angiograms were obtained that the neurosurgeon was able to determine that the AVM had indeed become amenable to microsurgery.

\section{Statistical Analysis}

Data are presented as mean and range for continuous variables and as frequency for categorical variables. Analysis was carried out using unpaired t-tests, Chisquare, and Fisher's exact tests, as appropriate. Assessment of means of more than 2 groups was carried out by ANOVA with post hoc analysis with Bonferroni correction as appropriate. Univariate analysis was used to test covariates predictive of perioperative complications. Interaction and confounding were assessed through stratification and relevant expansion covariates. Factors predictive in univariate analysis $(\mathrm{p}<0.15)^{1}$ were entered into a multivariate logistic regression analysis. Any $\mathrm{p}$ values of $\leq 0.05$ were considered statistically significant. Statistical analysis was carried out with Stata 10.0.

\section{Results}

Of the 264 patients included in the study, 120 (45\%) had an initial hemorrhagic presentation (Table 1). According to the SM grading scale for AVMs (Fig. 1 and Table 2), there were 27 SM Grade I lesions (10.2\%), 101 Grade II lesions (38.3\%), 96 Grade III lesions (36.4\%), 31 Grade IV lesions (11.7\%), and 9 Grade V lesions (3.4\%).

Among the 264 patients, 102 (38.6\%) had undergone prior endovascular embolization at our institution. The number of presurgical embolization sessions was 6 in 1 patient, 5 in 1 patient, 44 in 4 patients, 3 in 10 patients, 2 in 19 patients, and 1 in 67 patients. Additionally, 12 patients had a single Gamma Knife radiation procedure prior to surgery and 4 patients had a 2-stage treatment of Gamma Knife surgery. A total of 10 patients were treated with a combination of all treatment modalities.

Microsurgery resulted in permanent neurological

TABLE 1: Patient characteristics at initial presentation

\begin{tabular}{cc}
\hline Patient Characteristic $(n=264)$ & No. of Patients $(\%)$ \\
\hline sex & $140(53)$ \\
female & $124(47)$ \\
male & \\
smoker & $111(42)$ \\
yes & $153(58)$ \\
no & \\
hypertension & $83(31)$ \\
yes & $181(69)$ \\
no & \\
hyperlipidemia & $90(34)$ \\
yes & $174(66)$ \\
no & \\
hemorrhagic presentation & $120(45)$ \\
yes & $144(55)$ \\
no &
\end{tabular}

Neurosurg Focus / Volume 37 / September 2014 


\section{Spetzler-Martin Grading System: I-V Scale

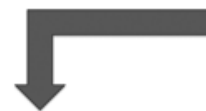 \\ CNS Location

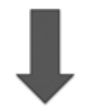 \\ Size

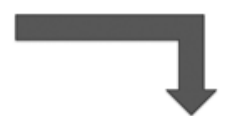 \\ Venous Drainage \\ Superficial 0 \\ Deep 1 \\ Medium 2 \\ (3-6 cm) \\ Large 3 \\ $(>6 \mathrm{~cm})$}

FIG. 1. AVMs are graded based on 3 parameters, illustrated with arrows (left to right): eloquence, size of the AVM nidus, and whether the AVM's venous drainage is superficial or deep. The grade is obtained by adding the points assigned from each category. A higher SM grade implies greater risk for postoperative morbidity and mortality.

deficits in 5 patients $(1.9 \%)$ and death in 7 patients $(2.7 \%)$. Permanent neurological deficits included 2 cases of hemiparesis, 1 of hemiplegia, 1 of aphasia, and 1 of vegetative state. All patients who died presented with a ruptured AVM and had poor findings on neurological examination upon presenting to the hospital prior to surgery. The characteristics and a detailed course of all patient mortalities are presented in Tables 4 and 5 .

Postoperative seizure was observed in 9 patients (3.4\%). Postoperative ventriculoperitoneal shunt placement was necessary in 8 patients (3\%). Five patients $(1.9 \%)$ developed postoperative infections that resolved without further complications. Of the 144 patients who presented with unruptured AVM, 10 experienced minor neurological deficits $(6.9 \%), 1$ experienced permanent neurological deficits $(0.69 \%)$, and 1 died $(0.69 \%)$. Five patients $(3.5 \%)$ experienced postoperative seizures, 2 required postoperative ventriculoperitoneal shunt placement (1.4\%), and 1 patient had a postoperative infection $(0.69 \%)$.

The majority of the treated unruptured AVMs were SM Grade III or lower, with 13 SM Grade I lesions (9.0\%), 66 Grade II lesions (45.8\%), and 50 Grade III lesions (34.8\%). A total of 15 high-grade unruptured AVMs-13 Grade IV $(9.0 \%)$ and 2 Grade V (1.4\%) - received intervention. Most of the unruptured AVMs were less than 6 $\mathrm{cm}$ in size, with 78 lesions (29.5\%) less than $3 \mathrm{~cm}$ and 62 lesions $(23.5 \%)$ ranging from 3 to $6 \mathrm{~cm}$ in size. The mRS score at discharge was 0.074 for patients presenting with unruptured AVM and 1.22 in patients presenting with hemorrhage. When results were stratified, $5.7 \%$ of patients without embolization had a complication versus $9.4 \%$ of patients with 1 or more embolizations. In multivariate analysis, predictors of complications (Table 3) were increasing AVM size (OR 3.2, 95\% CI 1.5-6.6; $\mathrm{p}=$ 0.001 ), increasing number of embolizations (OR 1.6, 95\% CI 1.1-2.2; $\mathrm{p}=0.01$ ), and unruptured AVMs (OR 2.7,95\% CI $1-7.2 ; \mathrm{p}=0.05)$.

Seven patients $(2.7 \%)$ who had undergone AVM embolization and 2 patients who had undergone Gamma Knife surgery experienced AVM rupture prior to microsurgical resection. The remaining course after surgery in
TABLE 2: Patient AVM characteristics according to SM grading scheme

\begin{tabular}{cc}
\hline SM Grade Component $(\mathrm{n}=264)$ & No. of Patients $(\%)$ \\
\hline SM size & $159(60.0)$ \\
small $(<3 \mathrm{~cm})$ & $95(36.0)$ \\
medium $(3-6 \mathrm{~cm})$ & $10(4.0)$ \\
large $(>6 \mathrm{~cm})$ & \\
SM location & $91(34.5)$ \\
noneloquent cortex & $173(65.5)$ \\
eloquent cortex & \\
SM venous drainage & $133(50.1)$ \\
superficial & $131(49.9)$ \\
deep & \\
SM Grade & $27(10.2)$ \\
I & $101(38.3)$ \\
II & $96(36.4)$ \\
III & $31(11.7)$ \\
IV & $9(3.4)$ \\
V
\end{tabular}

these patients was without further complications. In all patients, microsurgical resection resulted in complete obliteration and cure of the AVM. Upon discharge, 228 patients $(86.4 \%)$ had a favorable outcome (mRS score of $0-2)$. No patient had experienced hemorrhage after surgery. Of 264 patients, 244 (92.4\%) remained neurologically unchanged postoperatively.

\section{Discussion}

Treatment of cerebral AVMs undoubtedly remains one of the biggest challenges in the field of cerebrovascular neurosurgery. Advantages of surgery include quick elimination of the AVM in the majority of cases and significantly decreased or essentially nil risk of future hemorrhage. ${ }^{7}$ Furthermore, since the lesion is completely eliminated in the majority of cases, follow-up care is much less extensive than that required for less invasive treatment modalities.

Historically, surgical management of AVMs was reserved for patients with hemorrhagic presentation, intractable epilepsy, or progressively worsening neurological deficits. ${ }^{22,23}$ Early data, however, showed favorable results for surgical management of other AVM patients, such as those of Heros and $\mathrm{Tu},{ }^{13}$ who reported rates of surgical morbidity and mortality of $14.2 \%$ and $0 \%$, respectively, in 49 patients who presented with unruptured AVMs. Their results were equally favorable for 54 patients who pre-

TABLE 3: Patient outcomes: AVM microsurgery

\begin{tabular}{lccl}
\hline \multicolumn{1}{c}{ Predictor of Complications } & Odds Ratio & $95 \% \mathrm{Cl}$ & $\mathrm{p}$ value \\
\hline increasing no. of embolizations & 1.6 & $1.5-6.6$ & $<0.001$ \\
increasing AVM size & 3.2 & $1.2-2.2$ & $<0.01$ \\
unruptured AVM at presentation & 2.7 & $1.0-7.2$ & $<0.05$ \\
\hline
\end{tabular}


sented with ruptured AVMs (16.6\% morbidity rate and $1.6 \%$ mortality rate). Furthermore, more data became available on the dismal natural history of AVMs if they were left untreated. ${ }^{12,15}$ This new information suggested that a critical threshold exists wherein operative benefit outweighs operative risk and changes the natural history of the disease in carefully selected patients. This threshold was recognized by Spetzler and Martin ${ }^{22}$ and led to their creation of the most pertinent and effective grading scale for AVMs. It has been proven that this grading scale can be applied reliably to most AVMs with good agreement among observers. ${ }^{8}$

We presented data on a large single-center series of patients who underwent microsurgical resection of AVMs. Microsurgery resulted in death or permanent neurological deficits in only $4.6 \%$ of patients. From the deaths in this series (Tables 4 and 5), we can glean several important clinical insights. First and foremost, the possibility of AVM rupture is one that should receive great weight in counseling patients on the decision of if, how, and when is best to treat the AVM. In our series, all patients except for 1 were age 55 years or younger, and the majority were free of any major comorbidities. More than half of the patients had an AVM with a size of $3 \mathrm{~cm}$ or greater. A multimodality, minimally invasive approach of embolization and radiosurgery may perhaps be a safer means of curing a large AVM if it is detected in a timely manner. However, as these deaths illustrate, there is risk in using embolization to reduce AVM flow instead of volume, with a resulting possibility of short-term postembolization hemorrhage. All deaths were in patients who presented with hemorrhage and underwent urgent surgery. Therefore, the deaths of these patients also stand to remind the neurosurgical community that AVM detection prior to rupture remains a challenge, and a possible direction of future research is to elucidate which patients may be identified as candidates to screen for a cerebral AVM.

Over $92 \%$ of patients remained neurologically unchanged postoperatively. Also, $86.4 \%$ of patients had favorable clinical outcomes at discharge. Larger and unruptured AVMs, increasing number of embolizations, and unruptured presentation were identified as predictors of complications from operative excision.

\section{Unruptured AVM}

In our series, an unruptured AVM prior to microsurgery was a statistically significant predictor of postopera- tive complications. This is in agreement with the findings of Lawton et al., ${ }^{14}$ who used hemorrhagic presentation as a primary predictor variable in a consecutive series of 224 surgical AVM patients. In this group, hemorrhagic presentation was observed in 120 patients (54\%) and was associated with improved outcomes. A total of 15 patients (6.7\%) died. A key conclusion from their series is that hemorrhagic brain injury and its secondary effects may mask the surgical morbidity associated with microsurgery for AVMs. Sensitive measures of clinical outcomes, such as the mRS, are more likely to reveal slight changes in patients who initially were essentially without symptoms preoperatively. ${ }^{14}$ Therefore, in counseling patients with no history of hemorrhage or baseline deficits, it is important to note that even subtle postoperative deficits can be easily detected by the patient and clinician.

The majority of our patients who received operative intervention in the context of an unruptured AVM had SM Grade I-III lesions and were deemed good candidates for microsurgery, wherein the benefits outweighed the risks. In light of our favorable results, operative intervention in patients with unruptured AVMs should be seriously considered at high-volume experienced centers. The patients who can benefit the most are those who are determined to be good candidates for surgery and those who have lesions with characteristics and angioarchitecture that put them at high risk for rupture.

\section{Preoperative Embolization}

In the present study, 102 patients (38.6\%) had embolization procedures. Previous data highlight the favorable clinical outcomes of multimodality therapy for carefully selected patients. ${ }^{3,4,6,11,19}$ Presurgical embolization can safely decrease the size of AVMs and decrease the apparent risk of rupture in high-risk lesions such as highflow aneurysms or remote fistulas. ${ }^{28} \mathrm{Du}$ and colleagues ${ }^{9}$ have shown that diffuseness and deep perforating artery supply are subtle features of an AVM that predict worse outcomes after microsurgical resection, since deep perforators are friable, poorly visualized, and located in eloquent white matter tracts. ${ }^{9}$ Therefore, for microsurgery candidates, embolization is especially beneficial for elimination of deep feeding vessels. Furthermore, it is an important means by which operative blood loss can be greatly minimized. ${ }^{24,25}$

Despite the benefits of embolization, an increasing number of embolizations serve as a predictor of postoper-

TABLE 4: Deaths in a series of 264 patients who underwent microsurgical resection of AVM: AVM characteristics and embolization status prior to microsurgery

\begin{tabular}{ccccc}
\hline Patient No. & Age (yrs), Sex & Largest Diameter $(\mathrm{cm})$ & Eloquent Cortex & No. of Embolizations \\
\hline 1 & $38, \mathrm{M}$ & 3.5 & yes & none \\
2 & $55, \mathrm{M}$ & 2.0 & no & none \\
3 & $52, \mathrm{M}$ & 4.0 & yes & none \\
4 & $27, \mathrm{~F}$ & 2.5 & yes & none \\
5 & $47, \mathrm{M}$ & 3.0 & no & none \\
6 & $66, \mathrm{~F}$ & 4.0 & yes & 2 \\
7 & $43, \mathrm{M}$ & 3.5 & yes & 2 \\
\hline
\end{tabular}




\section{Microsurgical outcomes for AVMs}

TABLE 5: Deaths in a series of 264 patients who underwent microsurgical resection of AVM: preoperative, intraoperative, and postoperative findings*

\begin{tabular}{|c|c|c|c|}
\hline Patient No. & Preop Presentation & Op Remarks & Postop Findings \\
\hline 1 & GCS 4, ruptured AVM & $\begin{array}{l}\text { suboccipital craniectomy for evacuation of } \\
\text { cerebellar hematoma \& removal of AVM }\end{array}$ & $\begin{array}{l}\text { the patient became unstable postop \& soon thereafter } \\
\text { coded; aggressive resuscitation attempts were made but } \\
\text { were ineffective }\end{array}$ \\
\hline 2 & GCS 8, ruptured AVM & $\begin{array}{l}\text { rt frontotemporal craniotomy for evacuation } \\
\text { of hematoma \& subsequent excision of } \\
\text { frontal AVM }\end{array}$ & $\begin{array}{l}\text { the patient's course was complicated by multiple medi- } \\
\text { cal comorbidities: thrombocytopenia secondary to HIT, } \\
\text { alcoholic liver disease, aspiration pneumonia, sepsis, \& } \\
\text { It hemispheric seizure activity; eventually the decision to } \\
\text { withdraw care was made }\end{array}$ \\
\hline 3 & GCS 7, ruptured AVM & $\begin{array}{l}\text { rt hemicraniotomy for evacuation of hema- } \\
\text { toma \& parietal AVM }\end{array}$ & $\begin{array}{l}12 \text { hrs postop, patient had a sudden increase in intracranial } \\
\text { pressure; head CT showed evidence of a large epidural } \\
\text { hematoma, \& the patient was emergently taken back } \\
\text { to the operating room for evacuation of the hematoma; } \\
\text { postop, the patient remained neurologically devastated \& } \\
\text { coagulopathic, \& the family decided to withdraw care }\end{array}$ \\
\hline 4 & GCS 3, ruptured AVM & $\begin{array}{l}\text { It hemicraniotomy for decompression \& } \\
\text { evacuation of frontal hematoma \& AVM }\end{array}$ & $\begin{array}{l}\text { the patient did not improve postop; the family decided to } \\
\text { withdraw care }\end{array}$ \\
\hline 5 & GCS 4, ruptured AVM & $\begin{array}{l}\text { It hemicraniotomy for decompression of } \\
\text { It-sided hemisphere, evacuation of } \\
\text { hematoma, \& evacuation of partially } \\
\text { thrombosed AVM }\end{array}$ & $\begin{array}{l}\text { the patient did not improve postop \& progressed to brain } \\
\text { death }\end{array}$ \\
\hline 6 & GCS 7, ruptured AVM & $\begin{array}{l}\text { It frontotemporal craniotomy, evacuation of } \\
\text { hematoma, excision of It frontal AVM w/ } \\
\text { easily identifiable previously embolized } \mathrm{A}_{3} \\
\text { pedicle feeder }\end{array}$ & $\begin{array}{l}\text { patient's neurological exam failed to improve \& a massive } \\
\text { pulmonary embolism involving the main pulmonary } \\
\text { arteries complicated the course; the family decided to } \\
\text { withdraw care }\end{array}$ \\
\hline 7 & GCS 4, ruptured AVM & $\begin{array}{l}\text { It temporoparietal frontal craniotomy, evacu- } \\
\text { ation of hematoma, \& excision of complex } \\
\text { temporal AVM w/ MCA arterial feeders }\end{array}$ & $\begin{array}{l}2 \text { days status post } 2 \text { nd embolization, the AVM ruptured \& } \\
\text { the patient was taken for emergent surgery; postop, the } \\
\text { patient showed no signs of recovery \& the family opted } \\
\text { for withdrawal of care }\end{array}$ \\
\hline
\end{tabular}

* All deaths were in patients with prior hemorrhage who underwent an emergency operation. GCS = Glasgow Coma Scale; HIT = heparin-induced thrombocytopenia; MCA = middle cerebral artery.

ative complications, and the current data suggest the existence of a safety threshold for the number of embolization procedures. In the present series, patients who received embolization prior to surgery were initially deemed as high-risk candidates for open resection. Furthermore, it is possible that the patients receiving multiple embolization treatments were originally planned for curative treatment by a different modality, such as stereotactic radiosurgery. However, it was only after the course of successful and subsequent embolization that the patients could be reassessed and regarded as good surgical candidates with apparently small peri- and postoperative risk profiles. These findings are in line with those of Nataraj et al., ${ }^{19}$ who reported favorable outcomes in $86 \%$ of patients who underwent embolization and subsequent microsurgical resection versus $96.5 \%$ in those who underwent microsurgical resection alone. ${ }^{19}$

From the experience with the present series of patients, there are a few scenarios that make presurgical embolization potentially risky. First, there is the possibility of decreasing the flow too much in one area, resulting in increased flow in other areas of the nidus and/or cerebral vasculature. Second, diminishing venous outflow from embolization could cause AVM rupture. While embolization was a negative predictor of outcome in this series of patients, it was also likely a marker of patients with more complicated AVM angioarchitecture, which cannot be quantified or accounted for in a retrospective case series.

\section{AVM Size}

The present series was composed primarily of AVMs ranging from small to medium in size, and such lesions are known to be good candidates for microsurgical management. 18,22 This assessment is supported by the fact that Stuier and colleagues ${ }^{26}$ have shown that in patients harboring small- and medium-sized AVMs, dynamic autoregulatory function seems to be intact in the surrounding cerebrovascular bed perioperatively. ${ }^{26}$ Larger AVMs are also more likely to have more draining veins, which have also been shown to put a patient at risk for postoperative breakthrough complications. ${ }^{21}$ AVMs of SM Grade III or higher accounted for the majority of the complications noted in our series. Importantly, AVM size was a strong independent predictor of surgical complications. This finding emphasizes the importance of AVM size for stratification of surgical risk. 


\section{T. Theofanis et al.}

More data on microsurgery treatment in a larger group of cases with high SM grade are needed to elucidate specific components of the SM grading scale that can predict favorable operative outcomes in high-grade lesions. For giant cerebral AVMs located superficially or not involving critical components, resection can be expected to yield a good outcome. ${ }^{30}$ However, microsurgery of Grade IV-V AVMs based on the SM grading scale should be considered experimental. For these lesions, multimodality therapy may be considered an option that could provide more favorable outcomes. ${ }^{3,4}$

\section{Microsurgery and the ARUBA Trial}

Earlier this year, the ARUBA trial ${ }^{17}$ was published and the investigators reached a conclusion that medical management is superior to any intervention (microsurgery, embolization, or radiosurgery) in terms of patient outcomes. Such a study on the topic of AVMs has been long overdue, and we acknowledge the efforts of the authors. In the intervention group of the ARUBA trial, ${ }^{17} 5$ patients were treated with neurosurgical procedures, 30 with embolization, 31 with radiotherapy, and 28 with multimodality therapy.$^{17}$ The majority of patients in the ARUBA trial ${ }^{17}$ treatment arm received noninvasive and nonneurosurgical treatments (embolization and/or radiosurgery), neither of which is recognized by the neurosurgical community as a "cure" for AVMs in the truest sense of the word. In their eloquent response to the ARUBA trial, Russin and Spetzler ${ }^{20}$ noted that it remains difficult to resolve why only 5 patients received resection alone when 76 patients in the treatment arm had Grade I or II AVMs. ${ }^{20}$

Our data surpassed the results achieved in the interventional arm of the ARUBA trial. ${ }^{17}$ We demonstrated that with modern-day techniques and approaches, microsurgery can be undertaken with extremely low complication rates and provide excellent outcomes for patients. As such, we challenge the results of the ARUBA trial, ${ }^{17}$ wherein only 5 patients in the treatment arm received microsurgery, which is the only treatment that enables immediate cure and elimination of the lesion. The data we present are encouraging, and the presented outcomes are what should be expected at any high-volume neurovascular center. A trial comparing microsurgery and medical management is necessary to begin exploring the unanswered questions and clinical discrepancies raised by the ARUBA trial. ${ }^{17}$

\section{Limitations}

Key limiting factors of this study include the retrospective data analysis, single-center study, and recall bias. We recognize that our current results reflect those that should be expected from a high-volume neurovascular center.

\section{Conclusions}

Rapid advances in neurosurgical technical skills and tools, and the evolution from traditional surgery to microsurgery, have revolutionized the role of surgical intervention for AVMs.

\section{Disclosure}

Dr. Jabbour has been a consultant for ev3, Codman, and Mizuho. Dr. Tjoumakaris has been a consultant for Stryker. Dr. Rosenwasser has been a consultant for Boston Scientific. The other authors have no personal financial or institutional interest in any of the drugs, materials, or devices described in this article.

Author contributions to the study and manuscript preparation include the following. Conception and design: Tjoumakaris, Dalyai, Rosenwasser. Acquisition of data: Theofanis, Dalyai. Analysis and interpretation of data: Starke. Drafting the article: Tjoumakaris, Theofanis, Chalouhi, Dalyai, Starke, Rosenwasser. Critically revising the article: all authors. Reviewed submitted version of manuscript: all authors. Approved the final version of the manuscript on behalf of all authors: Tjoumakaris. Statistical analysis: Starke. Administrative/technical/material support: Tjoumakaris, Starke, Rosenwasser. Study supervision: Dalyai, Rosenwasser.

\section{References}

1. Altman DG: Practical Statistics for Medical Research. Boca Raton, FL: Chapman \& Hall/CRC, 1990

2. Brown RD Jr, Wiebers DO, Torner JC, O'Fallon WM: Frequency of intracranial hemorrhage as a presenting symptom and subtype analysis: a population-based study of intracranial vascular malformations in Olmsted Country, Minnesota. J Neurosurg 85:29-32, 1996

3. Chalouhi N, Dumont AS, Randazzo C, Tjoumakaris S, Gonzalez LF, Rosenwasser R, et al: Management of incidentally discovered intracranial vascular abnormalities. Neurosurg Focus 31(6):E1, 2011

4. Chang SD, Marcellus ML, Marks MP, Levy RP, Do HM, Steinberg GK: Multimodality treatment of giant intracranial arteriovenous malformations. Neurosurgery 53:1-13, 2003

5. Choi JH, Mast H, Hartmann A, Marshall RS, Pile-Spellman J, Mohr JP, et al: Clinical and morphological determinants of focal neurological deficits in patients with unruptured brain arteriovenous malformation. J Neurol Sci 287:126-130, 2009

6. Cockroft KM, Hwang SK, Rosenwasser RH: Endovascular treatment of cerebral arteriovenous malformations: indications, techniques, outcome, and complications. Neurosurg Clin N Am 16:367-380, x, 2005

7. Davies JM, Yanamadala V, Lawton MT: Comparative effectiveness of treatments for cerebral arteriovenous malformations: trends in nationwide outcomes from 2000 to 2009. Neurosurg Focus 33(1):E11, 2012

8. Du R, Dowd CF, Johnston SC, Young WL, Lawton MT: Interobserver variability in grading of brain arteriovenous malformations using the Spetzler-Martin system. Neurosurgery 57:668-675, 2005

9. Du R, Keyoung HM, Dowd CF, Young WL, Lawton MT: The effects of diffuseness and deep perforating artery supply on outcomes after microsurgical resection of brain arteriovenous malformations. Neurosurgery 60:638-648, 2007

10. Hartmann A, Mast H, Choi JH, Stapf C, Mohr JP: Treatment of arteriovenous malformations of the brain. Curr Neurol Neurosci Rep 7:28-34, 2007

11. Hartmann A, Mast H, Mohr JP, Pile-Spellman J, Connolly ES, Sciacca RR, et al: Determinants of staged endovascular and surgical treatment outcome of brain arteriovenous malformations. Stroke 36:2431-2435, 2005

12. Heros RC, Tu YK: Is surgical therapy needed for unruptured arteriovenous malformations? Neurology 37:279-286, 1987

13. Heros RC, Tu YK: Unruptured arteriovenous malformations: a dilemma in surgical decision making. Clin Neurosurg 33:187-236, 1986

14. Lawton MT, Du R, Tran MN, Achrol AS, McCulloch CE, Johnston SC, et al: Effect of presenting hemorrhage on outcome after microsurgical resection of brain arteriovenous malformations. Neurosurgery 56:485-493, 2005 


\section{Microsurgical outcomes for AVMs}

15. Mast H, Young WL, Koennecke HC, Sciacca RR, Osipov A, Pile-Spellman J, et al: Risk of spontaneous haemorrhage after diagnosis of cerebral arteriovenous malformation. Lancet 350:1065-1068, 1997

16. Meyer B, Schaller C, Frenkel C, Schramm J: Physiological steal around AVMs of the brain is not equivalent to cortical ischemia. Neurol Res 20 (Suppl 1):S13-S17, 1998

17. Mohr JP, Parides MK, Stapf C, Moquete E, Moy CS, Overbey JR, et al: Medical management with or without interventional therapy for unruptured brain arteriovenous malformations (ARUBA): a multicentre, non-blinded, randomised trial. Lancet 383:614-621, 2014

18. Morgan MK, Rochford AM, Tsahtsarlis A, Little N, Faulder KC: Surgical risks associated with the management of Grade I and II brain arteriovenous malformations. Neurosurgery 54:832-839, 2004

19. Nataraj A, Mohamed MB, Gholkar A, Vivar R, Watkins L, Aspoas R, et al: Multimodality treatment of cerebral arteriovenous malformations. World Neurosurg [epub ahead of print], 2013

20. Russin J, Spetzler R: Commentary: The ARUBA Trial. Neurosurgery 75:E96-E97, 2014 (Letter)

21. Schaller C, Urbach H, Schramm J, Meyer B: Role of venous drainage in cerebral arteriovenous malformation surgery, as related to the development of postoperative hyperperfusion injury. Neurosurgery 51:921-929, 2002

22. Spetzler RF, Martin NA: A proposed grading system for arteriovenous malformations. J Neurosurg 65:476-483, 1986

23. Stapf C, Mohr JP, Choi JH, Hartmann A, Mast H: Invasive treatment of unruptured brain arteriovenous malformations is experimental therapy. Curr Opin Neurol 19:63-68, 2006

24. Starke RM, Komotar RJ, Hwang BY, Fischer LE, Garrett MC, Otten ML, et al: Treatment guidelines for cerebral arteriovenous malformation microsurgery. Br J Neurosurg 23:376386,2009
25. Strozyk D, Nogueira RG, Lavine SD: Endovascular treatment of intracranial arteriovenous malformation. Neurosurg Clin N Am 20:399-418, 2009

26. Stüer C, Ikeda T, Stoffel M, Schaller C, Meyer B: Dynamic autoregulation testing does not indicate changes of cerebral blood flow before and after resection of small- and mediumsized cerebral AVM. Transl Stroke Res 2:60-66, 2011

27. Sturiale CL, Puca A, Calandrelli R, D’Arrigo S, Albanese A, Marchese E, et al: Relevance of bleeding pattern on clinical appearance and outcome in patients with hemorrhagic brain arteriovenous malformations. J Neurol Sci 324:118-123, 2013

28. Tjoumakaris SI, Jabbour PM, Gonzalez LF, Dumont AS, Randazzo CG, Rosenwasser RH: The evolution of future directions of neuroendovascular therapy: from clips to coils to? Clin Neurosurg 58:42-50, 2011

29. van Beijnum J, van der Worp HB, Buis DR, Al-Shahi Salman R, Kappelle LJ, Rinkel GJ, et al: Treatment of brain arteriovenous malformations: a systematic review and meta-analysis. JAMA 306:2011-2019, 2011

30. Zhao J, Yu T, Wang S, Zhao Y, Yang WY: Surgical treatment of giant intracranial arteriovenous malformations. Neurosurgery 67:1359-1370, 2010

Manuscript submitted May 5, 2014.

Accepted July 3, 2014.

Please include this information when citing this paper: DOI: 10.3171/2014.7.FOCUS14160.

Address correspondence to: Stavropoula Tjoumakaris, M.D., Department of Neurosurgery, Thomas Jefferson University Hospital, 909 Walnut St., Ste. 303, Philadelphia, PA 19107. email: stavropoula.tjoumakaris@jefferson.edu. 